\title{
Tail dependence and smoothness of time series
}

\author{
Helena Ferreira
}

Universidade da Beira Interior, Centro de Matemática e Aplicações (CMA-UBI), Avenida Marquês d'Avila e Bolama, 6200-001 Covilhã,

Portugal

helena.ferreira@ubi.pt

Marta Ferreira

Center of Mathematics of Minho University

Center for Computational and Stochastic Mathematics of University of Lisbon

Center of Statistics and Applications of University of Lisbon, Portugal msferreira@math.uminho.pt

\begin{abstract}
The risk of catastrophes is related to the possibility of occurring extreme values. Several statistical methodologies have been developed in order to evaluate the
\end{abstract}


propensity of a process for the occurrence of high values and the permanence of these in time. The extremal index $\theta$ (Leadbetter [16]) allows to infer the tendency for clustering of high values, but does not allow to evaluate the greater or less amount of oscillations in a cluster. The estimation of $\theta$ entails the validation of local dependence conditions regulating the distance between high levels oscillations of the process, which is difficult to implement in practice. In this work, we propose a smoothness coefficient to evaluate the degree of smoothness/oscillation in the trajectory of a process, with an intuitive reading and simple estimation. Application in some examples will be provided. We will see that, in a stationary sequence, it coincides with the tail dependence coefficient $\lambda$ (Sibuya [21], Joe [15]), providing a new interpretation of the latter. This relationship will inspire a new estimator for $\lambda$ and its performance will be evaluated based on a simulation study. We illustrate with an application to financial series.

Keywords: extreme values; smoothness coefficient; tail dependence coefficient.

AMS 2000 Subject Classification: $60 \mathrm{G} 70$

\section{Introduction}

The occurrence of high values in a stochastic process can mean a natural, social or economic catastrophe, which has motivated the development of statistical models and techniques for extremes of random variables (see, e.g., Gomes and Guillou, [12] and their references). The unpredictability we would like to dominate is based on the propensity of the process for high values and the mean time permanency of these, 
usually measured by the arithmetic inverse of the extremal index $\theta$ (Leadbetter [16]; Hsing et al. [14]). Clustering of high values can be predicted in stationary sequences that verify local dependency conditions $\mathrm{D}^{(k)}(u)$, which regulate the distance between oscillations of the process relative to high levels $u$ (Chernick et al. [2]). Under the validity of such conditions we can obtain expressions for the mean size $1 / \theta$ of a cluster of high values. Not only the validation of local dependence conditions is difficult in practice, but also the estimation of $1 / \theta$ does not give us information about the greater or less amount of oscillations in a cluster.

In this work, we propose a measure to distinguish between sequences with more oscillating trajectories from sequences with smoother ones, in what concerns the proportion of exceedances that are upcrossings. It has an intuitive reading, is easy to estimate and is free from validation of any conditions. This smoothness coefficient of a block of variables $\left\{X_{i}, n \leq i \leq m\right\}$ that we propose takes values in $[0,1]$ and grows with the degree of concordance of the variables.

By concordance we mean the concept in Joe [15], where we say that random variables $X_{1}, \ldots, X_{s}$ are more concordant than random variables $Y_{1}, \ldots, Y_{s}$, if $P\left(X_{1} \leq\right.$ $\left.x_{1}, \ldots, X_{s} \leq x_{s}\right) \geq P\left(Y_{1} \leq x_{1}, \ldots, Y_{s} \leq x_{s}\right)$ and $P\left(X_{1}>x_{1}, \ldots, X_{s}>x_{s}\right) \geq P\left(Y_{1}>\right.$ $\left.x_{1}, \ldots, Y_{s}>x_{s}\right)$, for all $x_{1}, \ldots, x_{s} \in(-\infty, \infty)$, that is, $X_{1}, \ldots, X_{s}$ are more likely than $Y_{1}, \ldots, Y_{s}$ to take on small values (and large values) simultaneously. For $s=2$ the two inequalities are equivalent.

We will apply it in theoretical examples. We will also verify that, in a stationary process, it coincides with the tail dependence coefficient $\lambda$ (Sibuya [21], Joe [15]), which gives us a new reading for this well-known coefficient in the literature of extremes. The new representation for the tail dependence coefficient inspires an estimation procedure that will be analysed through a simulation study. 
This paper is organized as follows: in Section 2 we introduce the smoothness coefficient and present some properties and examples. In Section 3 we consider a new estimator for $\lambda$ and analyse its performance through simulation. We illustrate with an application to financial series in Section 4.

\section{The smoothness coefficient}

Consider $\left\{X_{i}\right\}_{i \geq 1}$ a sequence of real random variables (r.v.) over the same probability space $(\Omega, \mathcal{A}, P)$ and denote $F_{i}$ the distribution function (d.f.) of $X_{i}, i \geq 1$. In the sequel we consider $F_{i}$ continuous for all $i \geq 1$. The assessment of the risk of occurrence of extreme phenomena is often associated with exceedances over high real thresholds by the variables in the sequence. Various examples and areas of application, such as finance, environment and actuarial science can be seen in, e.g., Maloney et al. [18] and references therein. A natural way to evaluate the propensity for oscillations within a process $\left\{X_{i}\right\}_{i \geq 1}$ is to compare the expected number of oscillations around the instant $i$,

$$
O_{i, j}=\left\{F_{i}\left(X_{i}\right) \leq u<F_{j}\left(X_{j}\right)\right\} \in \mathcal{A}, j=i-1, i+1,
$$

relative to real high levels $u$, with the expected number of exceedances of $u$,

$$
E_{i}=\left\{F_{j}\left(X_{j}\right)>u\right\} \in \mathcal{A}, j=i-1, i+1,
$$

around the instant $i$. Existing, at least, one exceedance between instants $n$ and $m$ $(n, m \in \mathbb{N})$, i.e., occurring $\left\{F_{j}\left(X_{j}\right)>u\right\}$ for some $j$ in $\{n, \ldots, m\}$, the expected total of oscillations will be closer of the expected total of exceedances, for $n \leq i \leq m$, in 
processes with more oscillating trajectories. We then propose as a summary measure of the result of this comparison between exceedances and oscillations, a coefficient with values in $[0,1]$, which increases with the concordance of the variables.

Definition 2.1. The smoothness coefficient $S_{n, m}$ of $\left\{X_{i}\right\}_{n \leq i \leq m}, 2 \leq n<m$, is defined by

$$
S_{n, m}=1-\lim _{u \uparrow 1} \frac{E\left(\sum_{i=n}^{m} \sum_{j \in V(i)} \mathbf{1}_{\left\{F_{i}\left(X_{i}\right) \leq u<F_{j}\left(X_{j}\right)\right\}} \mid \sum_{i=n}^{m} \mathbf{1}_{\left\{F_{i}\left(X_{i}\right)>u\right\}}>0\right)}{E\left(\sum_{i=n}^{m} \sum_{j \in V(i)} \mathbf{1}_{\left\{F_{j}\left(X_{j}\right)>u\right\}} \mid \sum_{i=n}^{m} \mathbf{1}_{\left\{F_{i}\left(X_{i}\right)>u\right\}}>0\right)},
$$

where $V(i)=\{i-1, i+1\}$, provided the limit exists.

Although expression (1) seems a little complex, it only translates the proportion of exceedances that are oscillations, around each location $i \in[n, m]$, given that there is at least one exceedance. In the following we present a result with a simpler alternative expression for coefficient $S_{n, m}$.

The proposed smoothness coefficient can naturally be expressed as a function of tail dependence coefficients

$$
\lambda(j \mid i)=\lim _{u \uparrow 1} P\left(F_{j}\left(X_{j}\right)>u \mid F_{i}\left(X_{i}\right)>u\right),
$$

which can also be written as

$$
\lambda(j \mid i)=1-\lim _{u \uparrow 1} \frac{u-d_{i, j}(u)}{1-u},
$$

where $d_{i, j}$ is the diagonal section of the copula of $\left(X_{i}, X_{j}\right)$. These coefficients summarize the behavior of the bivariate tails of a sequence and have been extensively studied and applied in the literature of extremes (see, e.g., Schmidt and Stadtmüller [20], Li [19], Ferreira and Ferreira [7], Lebedev [17], and references therein). 
Proposition 2.1. The smoothness coefficient $S_{n, m}$ of $\left\{X_{i}\right\}_{n \leq i \leq m}, 2 \leq n<m$, satisfies

$$
S_{n, m}=\frac{1}{m-n+1} \sum_{i=n}^{m} \frac{\lambda(i+1 \mid i)+\lambda(i \mid i-1)}{2},
$$

provided $\lambda(j \mid i)$ exists for all $n \leq i \leq m$ and $j=i-1, i+1$.

Proof. Observe that

$$
\begin{aligned}
S_{n, m} & =1-\lim _{u \uparrow 1} \frac{\sum_{i=n}^{m} \sum_{j \in V(i)}\left(P\left(F_{j}\left(X_{j}\right)>u\right)-P\left(F_{i}\left(X_{i}\right)>u, F_{j}\left(X_{j}\right)>u\right)\right)}{\sum_{i=n}^{m} \sum_{j \in V(i)} P\left(F_{j}\left(X_{j}\right)>u\right)} \\
& =\lim _{u \uparrow 1} \frac{\sum_{i=n}^{m} \sum_{j \in V(i)} P\left(F_{i}\left(X_{i}\right)>u, F_{j}\left(X_{j}\right)>u\right)}{2(m-n+1)(1-u)} \\
& =\frac{\sum_{i=n}^{m} \sum_{j \in V(i)} \lim _{u \uparrow 1} P\left(F_{j}\left(X_{j}\right)>u \mid F_{i}\left(X_{i}\right)>u\right)}{2(m-n+1)} .
\end{aligned}
$$

This result points to the reading of $\lambda=\lambda(j \mid i), j \in V(i)$, in a stationary process, as the smoothness coefficient for any block of variables $\left\{X_{i}, n \leq i \leq m\right\}$.

Corollary 2.2. If $\left\{X_{i}\right\}_{i \geq 1}$ is a stationary sequence with tail dependence coefficient $\lambda$, then $\lambda=S_{n, m}, 2 \leq n<m$.

Bivariate tail dependence increases with the concordance of the variables $(\mathrm{Li}$, [19]). We can therefore deduce the following properties from (3).

Proposition 2.3. Let the process $\left\{X_{i}\right\}_{n \leq i \leq m}$ have smoothness coefficient $S_{n, m}$, $2 \leq n<m$. Then

(i) $S_{n, m} \in[0,1]$; 
(ii) If $\left\{X_{i}, n \leq i \leq m\right\}$ are more concordant than $\left\{Y_{i}, n \leq i \leq m\right\}$, then $S_{n, m}^{(X)} \geq$ $S_{n, m}^{(Y)}$.

Proof. Result (i) is straightforward from the coefficient definition, since $P\left(F_{i}\left(X_{i}\right) \leq\right.$ $\left.u<F_{j}\left(X_{j}\right)\right) \leq P\left(F_{j}\left(X_{j}\right)>u\right)$. Assertion (ii) results from Proposition 2.1 and the fact that bivariate upper-tail dependence rises with concordance.

Remark 2.1. Observe that in Proposition 2.3 (ii) we only need the bivariate case of the "weak more concordant" condition $P\left(\bigcap_{i=n}^{m} F_{i}\left(X_{i}\right)>u\right) \geq P\left(\bigcap_{i=n}^{m} F_{i}\left(Y_{i}\right)>u\right)$.

For positive quadrant dependent variables in $\left\{X_{i}, n \leq i \leq m\right\}$, which is the case of max-stable sequences, in the bounds of the bivariate concordance relation, we have the independent and totally positive dependent variables. If all random pairs $\left\{\left(X_{i}, X_{j}\right)\right\}, j \in V(i), n \leq i \leq m$, are independent we have $S_{n, m}=0$, whereas if they are totally positive dependent then $S_{n, m}=1$.

In the context of max-stable sequences, the independence or total bivariate dependence of the variables in $\left\{X_{i}, n \leq i \leq m\right\}$ is equivalent to the independence or total dependence of all variables. Thus, if $\left\{X_{i}\right\}_{i \geq 1}$ is max-stable, then for $2 \leq n<m$, we will have $S_{n, m}=0$ if and only if $X_{i}, n \leq i \leq m$, are independent and $S_{n, m}=1$ if and only if $X_{i}, n \leq i \leq m$, are totally dependent. For the context of max-stability, we also have the possibility of relating $S_{n, m}$ with the extremal coefficients $\epsilon$ (Tiago de Oliveira [23], Smith [22]), which allows the estimation of the coefficients $\lambda(j \mid i)$ by estimating expected values (Ferreira, [10]).

Example 2.1. Consider the r-factor model (Einmahl et al. [4])

$$
X_{n}=\max _{s=1, \ldots, r} a_{s, n}^{\alpha} Z_{s}^{\alpha}, n \geq 1
$$


where factors $Z_{s}, s=1, \ldots, r$, are independent and Fréchet $(\alpha)$ distributed r.v., $\alpha>$ 0 , and $\left\{a_{s, n}, s=1, \ldots, r\right\}_{n \geq 1}$ are non-negative constants such that $\sum_{s=1}^{r} a_{s, n}>$ 0. Variables in $\left\{X_{n}\right\}_{n \geq 1}$ are not identically distributed since each one of the $r$ factors $Z_{1}, \ldots, Z_{r}$ contribute to the value of $X_{n}$ with weights $a_{s, n}$ updated over time n. Specifically we have

$$
F_{n}(x)=\exp \left(-x^{-1} \sum_{s=1}^{r} a_{s, n}^{\alpha}\right), x>0, n \geq 1
$$

We have

$$
\begin{aligned}
\lambda(j \mid i) & =\lim _{u \uparrow 1} \frac{P\left(F_{i}\left(X_{i}\right)>u, F_{j}\left(X_{j}\right)>u\right)}{P\left(F_{i}\left(X_{i}\right)>u\right)} \\
& =2-\lim _{u \uparrow 1} \frac{1-P\left(F_{i}\left(X_{i}\right) \leq u, F_{j}\left(X_{j}\right) \leq u\right)}{1-u} .
\end{aligned}
$$

Observe that

$$
\begin{aligned}
P\left(F_{i}\left(X_{i}\right) \leq u, F_{j}\left(X_{j}\right) \leq u\right) & =P\left(X_{i} \leq 1 /\left(-\ln u\left(\sum_{s=1}^{r} a_{s, i}^{\alpha}\right)^{-1}\right), X_{j} \leq 1 /\left(-\ln u\left(\sum_{s=1}^{r} a_{s, j}^{\alpha}\right)^{-1}\right)\right) \\
& =P\left(\bigcap_{s=1}^{r} Z_{s}^{\alpha} \leq \min \left(a_{s, i}^{-\alpha} /\left(-\ln u\left(\sum_{s=1}^{r} a_{s, i}^{\alpha}\right)^{-1}\right), a_{s, j}^{-\alpha} /\left(-\ln u\left(\sum_{s=1}^{r} a_{s, j}^{\alpha}\right)^{-1}\right)\right)\right)
\end{aligned}
$$

Thus, for the dependence on the tail of $X_{i}$ and $X_{j}$, we have

$$
\begin{aligned}
\lambda(j \mid i) & =2-\lim _{u \uparrow 1} \frac{1-u^{\sum_{s=1}^{r} \max \left(a_{s, i}^{\alpha} / \sum_{s=1}^{r} a_{s, i}^{\alpha}, a_{s, j}^{\alpha} / \sum_{s=1}^{r} a_{s, j}^{\alpha}\right)}}{1-u} \\
& =2-\sum_{s=1}^{r} \max \left(a_{s, i}^{\alpha} / \sum_{s=1}^{r} a_{s, i}^{\alpha}, a_{s, j}^{\alpha} / \sum_{s=1}^{r} a_{s, j}^{\alpha}\right) .
\end{aligned}
$$


Denoting $b_{s, n}=a_{s, n}^{\alpha} / \sum_{s=1}^{r} a_{s, n}^{\alpha}, n \geq 1, s=1, \ldots, r$, we have

$$
\begin{aligned}
S_{n, m} & =\frac{1}{2(m-n+1)} \sum_{i=n}^{m}\left(2-\sum_{s=1}^{r} \max \left(b_{s, i-1}, b_{s, i}\right)+2-\sum_{s=1}^{r} \max \left(b_{s, i}, b_{s, i+1}\right)\right) \\
& =2-\frac{1}{2(m-n+1)} \sum_{i=n}^{m} \sum_{s=1}^{r}\left(\max \left(b_{s, i-1}, b_{s, i}\right)+\max \left(b_{s, i}, b_{s, i+1}\right)\right) .
\end{aligned}
$$

Some intuitive cases present maximum or minimum smoothness. In the particular case of $a_{s, n}=a_{s}$, for all $n \geq 1$, we have a constant sequence and $b_{s, n}=b_{s}$, for all $n \geq 1$. Thus we obtain $\sum_{s=1}^{r} b_{s}=1$ and $S_{n, m}=1$.

If $r=1$, then $\left\{X_{n}\right\}_{n \geq 1}$ is a sequence of totally dependent variables and we have $\lambda(j \mid i)=1$ and $S_{n, m}=1$.

Under the special case of equally weighted factors, that is, $a_{s, n}=a_{n}, s=1, \ldots, r$, $n \geq 1$, and $X_{n}=a_{n}^{\alpha} \max _{s=1, \ldots, r} Z_{s}^{\alpha}$, we have

$$
b_{s, n}=b_{n} \equiv \frac{a_{n}^{\alpha}}{r a_{n}^{\alpha}}=\frac{1}{r}, s=1, \ldots, r, n \geq 1
$$

and therefore

$$
\begin{aligned}
S_{n, m} & =2-\frac{1}{2(m-n+1)} \sum_{i=n}^{m} r\left(\max \left(b_{i-1}, b_{i}\right)+\max \left(b_{i}, b_{i+1}\right)\right) \\
& =2-\frac{1}{2(m-n+1)} \sum_{i=n}^{m} r\left(\frac{2}{r}\right)=1 .
\end{aligned}
$$

If we consider $\alpha=1, r=2$ and $a_{1, i}=1=1-a_{2, i}$ for odd $i$ and $a_{1, i}=0=1-a_{2, i}$ for even $i$, we obtain $S_{n, m}=0$, for all $n$ and $m, 2 \leq n<m$.

Example 2.2. (Temporary Failures Model) Let $\left\{Y_{n}\right\}_{n \geq 1}$ be a sequence of independent and identically distributed (i.i.d.) variables and independent of the sequence of 
Bernoulli variables $\left\{Z_{n}\right\}_{n \geq 1}$. Consider notations $F(x)=P\left(Y_{n} \leq x\right), n \geq 1$, and

$$
p_{n, n+1, \ldots, n+s}\left(i_{0}, i_{1}, \ldots, i_{s}\right)=P\left(Z_{n}=i_{0}, Z_{n+1}=i_{1}, \ldots, Z_{n+s}=i_{s}\right),
$$

$i_{0}, \ldots, i_{s} \in\{0,1\}, s \geq 1$. We denominate by temporary failures model, a sequence $\left\{X_{n}\right\}_{n \geq 1}$ defined as follows:

$$
\begin{aligned}
& X_{1}=Y_{1} \\
& X_{n}=\left\{\begin{array}{ll}
X_{n-1}, & \text { se } Z_{n}=0 \\
Y_{n} & , \text { se } Z_{n}=1
\end{array}, n \geq 2 .\right.
\end{aligned}
$$

Such designation relies on the interpretation of $\left\{Z_{n}\right\}_{n \geq 1}$ as a sequence of states corresponding to the registration or non-registration of values of $\left\{X_{n}\right\}_{n \geq 1}$. Thus, if for example, $\left\{Z_{1}=1, Z_{2}=0, Z_{3}=0, Z_{4}=1, Z_{5}=1, Z_{6}=0, Z_{7}=1, Z_{8}=\right.$ $\left.0, Z_{9}=0, Z_{10}=0, Z_{11}=1\right\}$, we will have, almost surely, $\left\{X_{1}=Y_{1}, X_{2}=Y_{1}, X_{3}=\right.$ $\left.Y_{1}, X_{4}=Y_{4}, X_{5}=Y_{5}, X_{6}=Y_{5}, X_{7}=Y_{7}, X_{8}=Y_{7}, X_{9}=Y_{7}, X_{10}=Y_{7}, X_{11}=Y_{11}\right\}$. Zero sequences at the values of $\left\{Z_{n}\right\}_{n \geq 1}$ determine replicates of the last recorded value of $\left\{Y_{n}\right\}_{n \geq 1}$. If $n$ is the time, the zeros of $Z_{n}$ mean a stop of the register in time, keeping the last record. Let us consider a short-failures model to illustrate the smoothness coefficient calculation. In the short-failures model, we assume that $p_{n, n+1}(0,0)=0$, i.e., it is almost impossible to lose two or more consecutive records of $\left\{Y_{n}\right\}_{n \geq 1}$. 
We start by deriving the common d.f. of $X_{n}$ :

$$
\begin{aligned}
P\left(X_{n} \leq x\right) & =P\left(X_{n-1} \leq x, Z_{n}=0\right)+P\left(Y_{n} \leq x, Z_{n}=1\right) \\
& =F(x) p_{n-1, n}(1,0)+F(x) p_{n}(1) \\
& =F(x) p_{n}(0)+F(x) p_{n}(1) \\
& =F(x) .
\end{aligned}
$$

For $u \in(0,1]$ and $v=F^{-1}(u)$, where $F^{-1}$ is the inverse function of $F$, we have

$$
\begin{aligned}
& P\left(F\left(X_{i}\right) \leq u, F\left(X_{i+1}\right) \leq u\right) \\
= & P\left(X_{i} \leq v, X_{i+1} \leq v, Z_{i}=1, Z_{i+1}=1\right) \\
& +P\left(X_{i} \leq v, X_{i+1} \leq v, Z_{i}=1, Z_{i+1}=0\right) \\
& +P\left(X_{i} \leq v, X_{i+1} \leq v, Z_{i}=0, Z_{i+1}=1\right) \\
= & F^{2}(v) p_{i, i+1}(1,1)+F(v) p_{i, i+1}(1,0)+F^{2}(v) p_{i-1, i, i+1}(1,0,1) \\
= & u^{2}\left(p_{i, i+1}(1,1)+p_{i, i+1}(0,1)\right)+u p_{i, i+1}(1,0) .
\end{aligned}
$$

Therefore,

$$
\begin{aligned}
\lambda(i+1 \mid i) & =2-\lim _{u \uparrow 1} \frac{1-P\left(F\left(X_{i}\right) \leq u, F\left(X_{i+1}\right) \leq u\right)}{1-u} \\
& =2-\lim _{u \uparrow 1} \frac{1-u^{2}\left(p_{i, i+1}(1,1)+p_{i, i+1}(0,1)\right)-u p_{i, i+1}(1,0)}{1-u} \\
& =2-2\left(p_{i, i+1}(1,1)+p_{i, i+1}(0,1)\right)-p_{i, i+1}(1,0) \\
& =2-2\left(1-p_{i, i+1}(1,0)\right)-p_{i, i+1}(1,0) \\
& =p_{i, i+1}(1,0),
\end{aligned}
$$


and we obtain the smoothness coefficient given by

$$
\begin{aligned}
S_{n, m} & =\frac{1}{2(m-n+1)} \sum_{i=n}^{m}\left(p_{i-1, i}(1,0)+p_{i, i+1}(1,0)\right) \\
& =\frac{1}{2(m-n+1)} \sum_{i=n}^{m}\left(p_{i}(0)+p_{i+1}(0)\right) .
\end{aligned}
$$

We can see that $S_{n, m}$ increases with the tendency to stop in the initial sequence records, as expected. With some more time-consuming calculations, we can extend the result to models with longer lasting failures.

We note that in this short-failures model, the estimation of $p_{i, i+1}(1,0)$ allows us to estimate $S_{n, m}$. The estimation of $p_{i, i+1}(1,0)$ can be done from the natural estimation of $P\left(X_{i}=X_{i+1}\right)=E\left(\mathbf{1}_{\left\{X_{i}=X_{i+1}\right\}}\right)$, since, in general, $\left\{Y_{n}\right\}_{n \geq 1}$ and $\left\{Z_{n}\right\}_{n \geq 1}$ are unobservable sequences.

\section{A new estimator for $\lambda$ under stationarity}

The usual linear Pearson's correlation coefficient does not give us enough insight about the amount of dependence in the tails (Embrechts et al. [5]). Extreme values theory is the natural framework to address this topic. The tail dependence coefficient $\lambda$ is perhaps the most common measure of extremal dependency. Many other coefficients have been presented in the literature, most of them related to $\lambda$ (see, e.g., Schmidt and Stadtmüller [20], Li [19], Ferreira and Ferreira [8], and references therein). The smoothness coefficient introduced here is another measure of tail dependence and from Corollary 2.2 it coincides with $\lambda$ under stationarity. Inference based on the definition in (1) is quite straightforward by taking the respective empirical counterparts. Thus, we can state a new estimator for $\lambda$ based on $S_{n, m}$, which we denote $\hat{\lambda}^{F F}$. More precisely, considering a stationary sequence $\left\{X_{n}\right\}_{n \geq 1}$ with 
marginal d.f. $F$ and $U(u)$ and $E(u)$, respectively the number of upcrossings and the number of exceedances of a high level $u$ of $\left\{F\left(X_{n}\right)\right\}_{n \geq 1}$, we have

$$
\hat{\lambda}^{F F}:=\hat{S}_{n, m}=1-\frac{U(u)}{E(u)} .
$$

In the following we address a simulation study in order to analyse the performance of $\hat{\lambda}^{F F}$ in (4). We also consider two estimators of $\lambda$ well-known and commonly used in literature, motivated by (2):

$$
\hat{\lambda}^{L O G}:=2-\frac{\log \widehat{d}_{i, j}(u)}{\log u} \quad \text { and } \quad \hat{\lambda}^{S E C}:=2-\frac{1-\widehat{d}_{i, j}(u)}{1-u},
$$

where

$$
\widehat{d}_{i, j}(u):=\frac{1}{n-1} \sum_{i=1}^{n-1} \mathbf{1}_{\left\{\hat{F}\left(X_{i}\right) \leq u, \hat{F}\left(X_{i+1}\right) \leq u\right\}},
$$

and $\hat{F}$ corresponds to the empirical d.f. of $F$. See Frahm et al. [11] and references therein.

The simulations correspond to 200 replicas of samples with size $n=1000$ and $n=5000$ from the following models for $\left\{X_{n}\right\}_{n \geq 1}$ :

- First-order max-autoregressive (Davis and Resnick [3]) denoted MAR(1): $X_{n}=\max \left(c X_{n-1},(1-c) Z_{n}\right), 0<c<1$, with $\left\{Z_{n}\right\}_{n \geq 1}$ a sequence of i.i.d. r.v. with unit Fréchet d.f., as well as $X_{0}$ and thus $X_{n}, n \geq 1$. We have $\lambda=c$ (see, e.g., Ferreira and Ferreira [6]);

- First order moving-maximum (Davis and Resnick [3]) denoted MMA(1): $X_{n}=\max \left(c Z_{n},(1-c) Z_{n-1}\right), 0<c<1$, with $\left\{Z_{n}\right\}_{n \geq 1}$ a sequence of i.i.d. r.v. with unit Fréchet d.f., as well as $Z_{0}$ and thus $X_{n}, n \geq 1$. We have $\lambda=$ $\max (c, 1-c)$ (see, e.g., Heffernan et al. [13]); 
- First order autoregressive Yeh-Arnold-Robertson Pareto(III) (Arnold [1]), denoted $\operatorname{YARP}(1)$ :

$X_{n}=\min \left(p^{-1 / \alpha} X_{n-1}, \frac{1}{1-U_{n}} \varepsilon_{n}\right)$, where $\left\{\varepsilon_{n}\right\}_{n \geq 1}$, is a sequence of i.i.d. r.v. coming from a Pareto(III) $(0, \sigma, \alpha)$, i.e., $1-F_{X}(x)=\left[1+(x / \sigma)^{\alpha}\right]^{-1}, \sigma, \alpha>0$ and sequence $\left\{U_{n}\right\}_{n \geq 1}$ of i.i.d. r.v. coming from $\operatorname{Bernoulli}(p), 0<p<1$, independent of $\varepsilon_{n}, n \geq 1$. We consider $1 / 0 \equiv+\infty, X_{0} \frown$ Pareto(III) $(0, \sigma, \alpha)$ and thus $X_{n}, n \geq 1$. We have $\lambda=p$ (Ferreira [9]).

The absolute bias (abias) and the root mean squared error (rmse) derived from simulations are in Tables 1 and 2, where we considered the high level $u$ given by the 95\% sample quantile. Quantiles 90\% and 99\% were also used but do not improve the results and are not reported. The values in bold correspond to the least absolute bias and the least root mean squared error obtained in each model. We can see that the three estimators have very similar performances. The estimator $\hat{\lambda}^{F F}$ proposed here, being of very simple application, thus constitutes a possible alternative.

\section{Application to financial data}

Financial investors are very interested in the risk assessment of stock markets. It is commonly accepted that log-differences of index prices (log-returns) constitute a stationary sequence. Markets that exhibit greater volatility or variability in a short period of time represent greater risk. Hence volatility is of great interest to investors. One way to assess volatility is through the absolute value of log-returns. In the analysis that we are going to present, we consider the daily closing log-returns of the following stock market indexes: DJI, S\&P500 and FTSE100. We are going to apply estimators $\hat{\lambda}^{L O G}, \hat{\lambda}^{S E C}$ and $\hat{\lambda}^{F F}$, considering the $95 \%$ sample quantile, 
Table 1: Simulation results corresponding to the absolute bias (abias) and root mean squared error (rmse) obtained for estimators $\hat{\lambda}^{F F}, \hat{\lambda}^{L O G}$ and $\hat{\lambda}^{S E C}$, considering $u$ the $95 \%$ sample quantile and $n=1000$, within models $\operatorname{MAR}(1)$ with parameter values $c=0.25,0.5,0.75, \operatorname{MMA}(1)$ with parameter values $c=0.25,0.5,0.75$ and $\operatorname{YARP}(1)$ parameter values $p=0.25,0.5,0.75$.

\begin{tabular}{|c|c|c|c|c|c|c|c|}
\hline & \multirow[b]{2}{*}{$n=1000$} & \multicolumn{2}{|c|}{$\hat{\lambda}^{F F}$} & \multicolumn{2}{|c|}{$\hat{\lambda}^{L O G}$} & \multicolumn{2}{|c|}{$\hat{\lambda}^{S E C}$} \\
\hline & & abias & rmse & abias & rmse & abias & rmse \\
\hline \multirow{3}{*}{$\operatorname{MAR}(1)$} & $c=0.25$ & 0.0559 & 0.0723 & 0.0579 & 0.0745 & 0.0566 & 0.0724 \\
\hline & $c=0.50$ & 0.0556 & 0.0695 & 0.0557 & 0.0680 & 0.0561 & 0.0700 \\
\hline & $c=0.75$ & 0.0457 & 0.0550 & 0.0489 & 0.0594 & 0.0456 & 0.0551 \\
\hline \multirow{3}{*}{ MMA(1) } & $c=0.25$ & 0.0163 & 0.022 & 0.0198 & 0.0257 & 0.0277 & 0.0354 \\
\hline & $c=0.50$ & 0.0453 & 0.0581 & 0.0430 & 0.0533 & 0.0461 & 0.0587 \\
\hline & $c=0.75$ & 0.0439 & 0.0523 & 0.0348 & 0.044 & 0.0440 & 0.0527 \\
\hline \multirow{3}{*}{$\operatorname{YARP}(1)$} & $p=0.25$ & 0.0520 & 0.0678 & 0.0531 & 0.0695 & 0.0524 & 0.0678 \\
\hline & $p=0.50$ & 0.0576 & 0.0695 & 0.0503 & 0.0623 & 0.0577 & 0.0699 \\
\hline & $p=0.75$ & 0.0469 & 0.0604 & 0.0485 & 0.0633 & 0.0471 & 0.0604 \\
\hline
\end{tabular}

to each financial time series covering two one-year periods: 2015 and 2017 (Figure

1). So, roughly speaking, we can say that we will evaluate the behavior of financial markets in two periods $[n, m]$, chosen by us, before and after the Brexit referendum. Recalling the definition of the smoothness coefficient, it is not the magnitude of the oscillations that is being evaluated, but rather the greater or lesser number of oscillations, that is, the tendency of the trajectory to oscillate. The results are given in Table 3. They correspond to little soft trajectories and there is some decrease in smoothness, from 2015 to 2017, in DJI and S\&P500. According to the values 
Table 2: Simulation results corresponding to the absolute bias (abias) and root mean squared error (rmse) obtained for estimators $\hat{\lambda}^{F F}, \hat{\lambda}^{L O G}$ and $\hat{\lambda}^{S E C}$, considering $u$ the $95 \%$ sample quantile and $n=5000$, within models $\operatorname{MAR}(1)$ with parameter values $c=0.25,0.5,0.75, \operatorname{MMA}(1)$ with parameter values $c=0.25,0.5,0.75$ and $\operatorname{YARP}(1)$ parameter values $p=0.25,0.5,0.75$.

\begin{tabular}{|c|c|c|c|c|c|c|c|}
\hline & \multirow[b]{2}{*}{$n=5000$} & \multicolumn{2}{|c|}{$\hat{\lambda}^{F F}$} & \multicolumn{2}{|c|}{$\hat{\lambda}^{L O G}$} & \multicolumn{2}{|c|}{$\hat{\lambda}^{S E C}$} \\
\hline & & abias & rmse & abias & rmse & abias & rmse \\
\hline \multirow{3}{*}{$\operatorname{MAR}(1)$} & $c=0.25$ & 0.0316 & 0.0406 & 0.0307 & 0.0390 & 0.0316 & 0.0405 \\
\hline & $c=0.50$ & 0.0338 & 0.0414 & 0.0238 & 0.0301 & 0.0339 & 0.0415 \\
\hline & $c=0.75$ & 0.0218 & 0.0279 & 0.0224 & 0.0285 & 0.0218 & 0.0279 \\
\hline \multirow{3}{*}{ MMA(1) } & $c=0.25$ & 0.0180 & 0.0197 & 0.0066 & 0.0090 & 0.0205 & 0.0221 \\
\hline & $c=0.50$ & 0.0325 & 0.0381 & 0.0183 & 0.0229 & 0.0326 & 0.0382 \\
\hline & $c=0.75$ & 0.0321 & 0.0376 & 0.0183 & 0.0235 & 0.0320 & 0.0376 \\
\hline \multirow{3}{*}{$\operatorname{YARP}(1)$} & $p=0.25$ & 0.0298 & 0.0372 & 0.0262 & 0.0316 & 0.0299 & 0.0373 \\
\hline & $p=0.50$ & 0.0383 & 0.0445 & 0.0218 & 0.0281 & 0.0385 & 0.0446 \\
\hline & $p=0.75$ & 0.0226 & 0.0286 & 0.0228 & 0.0282 & 0.0228 & 0.0287 \\
\hline
\end{tabular}

obtained for the estimates of the proposed coefficient, we can say that the volatilities of the DJI and S\&P500 indices show more fluctuations in 2017 than in 2015. The volatilities of the FTSE100 index do not present great differences in the intensity of the fluctuations, when measured by this coefficient. 
Table 3: Estimates of $\hat{\lambda}^{L O G}, \hat{\lambda}^{S E C}$ and $\hat{\lambda}^{F F}$, considering the $95 \%$ sample quantile, obtained from volatility of the indexes DJI, S\&P500 and FTSE100, in years 2015 and 2017.

\begin{tabular}{|c|c|c|c|c|c|c|c|c|}
\hline DJI & 2015 & 2017 & S\&P500 & 2015 & 2017 & FTSE & 2015 & 2017 \\
\hline$\hat{\lambda}^{L O G}$ & 0.3607 & 0.1105 & $\hat{\lambda}^{L O G}$ & 0.2677 & 0.0241 & $\hat{\lambda}^{L O G}$ & 0.2116 & 0.2041 \\
\hline$\hat{\lambda}^{S E C}$ & 0.3871 & 0.1526 & $\hat{\lambda}^{S E C}$ & 0.2996 & 0.0723 & $\hat{\lambda}^{S E C}$ & 0.2470 & 0.2400 \\
\hline$\hat{\lambda}^{F F}$ & 0.2308 & 0.0769 & $\hat{\lambda}^{F F}$ & 0.3077 & 0.0000 & $\hat{\lambda}^{F F}$ & 0.2308 & 0.1538 \\
\hline
\end{tabular}

\section{Conclusions}

The tail dependence coefficient is one of the most well-known measures used in the evaluation of the risk of extreme observations. This work reinforces the importance and preference found by the use of this measure when relating it to the degree of oscillations in the trajectory of a process. This aspect is particularly useful in the evaluation of risk in financial, environmental, and other series. The contribution of this work shows that there is still a way of researching around this coefficient and the importance of investing in its estimation.

\section{Acknowledgements}

The authors thank the reviewers and the associated editor for the important and valuable comments that contributed to the improvement of this work.

The first author was partially supported by the research unit Centre of Mathematics and Applications of University of Beira Interior UIDB/00212/2020 - FCT (Fundação para a Ciência e a Tecnologia). The second author was financed by Portuguese Funds through FCT - Fundação para a Ciência e a Tecnologia within the Projects UIDB/00013/2020 and UIDP/00013/2020 of Centre of Mathematics of the University of Minho, UIDB/00006/2020 of Centre of Statistics and its Applications 
of University of Lisbon and PTDC/MAT-STA/28243/2017.

\section{References}

[1] Arnold, B.C. (2001). Pareto Processes. In: Handbook of Statistics (D.N. Shanbhag and C.R. Rao, eds.), Vol. 19, Elsevier Science B.V.

[2] Chernick M.R., Hsing T., McCormick W.P. (1991). Calculating the extremal index for a class of stationary sequences. Advances in Applied Probability 23, $835-850$.

[3] Davis, R., Resnick, S. (1989) Basic properties and prediction of max-ARMA processes. Adv. Appl. Probab. 21, 781-803.

[4] Einmahl, J. H. J., Krajina, A., Segers, J. (2012). An M-estimator for tail dependence in arbitrary dimensions. The Annals of Statistics, 40(3), 1764-1793.

[5] Embrechts, P., McNeil, A., Straumann, D. (2002). Correlation and dependence in risk management: properties and pitfalls. In "Risk Management: Value at Risk and Beyond" (M.A.H. Dempster, Eds.), Cambridge University Press, Cambridge, $176-223$.

[6] Ferreira, M., Ferreira, H. (2012). On extremal dependence: some contributions. TEST 21(3), 566-583.

[7] Ferreira, H., Ferreira, M. (2014) Extremal behavior of pMAX processes. Statistics and Probability Letters, 93, 46-57.

[8] Ferreira, H., Ferreira, M. (2018) Multidimensional extremal dependence coefficients. Statistics \& Probability Letters 133, 1-8. 
[9] Ferreira, M. (2012). On the extremal behavior of a pareto process: an alternative for armax modeling. Kybernetika 48(1), 31-49.

[10] Ferreira, M. (2013) Nonparametric estimation of the tail-dependence coefficient. RevStat, 11(1), 1-16.

[11] Frahm, G., Junker, M., Schmidt, R. (2005) Estimating the tail-dependence coefficient: Properties and pitfalls. Insurance: Mathematics and Economics, 37, $80-100$.

[12] Gomes, M.I., Guillou, A. (2015) Extreme Value Theory and Statistics of Univariate Extremes: A Review. International Statistical Review, 83, 263-292.

[13] Heffernan, J.E., Tawn, J.A., Zhang, Z. (2007). Asymptotically (in)dependent multivariate maxima of moving maxima processes. Extremes 10(1-2), 57-82.

[14] Hsing, T., Hüsler, J., Leadbetter, M.R. (1988) On the exceedance point process for a stationary sequence. Probab. Th. Rel. Fields, 78, 97-112.

[15] Joe, H. (1997) Multivariate Models and Dependence Concepts. Monographs on Statistics and Applied Probability 73, Chapman and Hall, London.

[16] Leadbetter M.R. (1983). Extremes and local dependence in stationary processes. Z. Wahrscheinlichkeitstheor Verw. Geb., 65, 291-306.

[17] Lebedev, A.V. (2019). On the Interrelation between Dependence Coefficients of Bivariate Extreme Value Copulas. Markov Processes and Related Fields, 25, $639-648$

[18] Moloney, N.R., Faranda, D., Sato, Y. (2019). An overview of the extremal index. Chaos, 29(2), 022101.

[19] Li, H. (2009) Orthant tail dependence of multivariate extreme value distributions. Journal of Multivariate Analysis, 100, 243-256. 
[20] Schmidt R., Stadtmüller U. (2006) Non-parametric Estimation of Tail Dependence. Scandinavian Journal of Statistics, 33, 307-335.

[21] Sibuya, M. (1960) Bivariate extreme statistics. Ann. Inst. Statist. Math., 11, $195-210$.

[22] Smith, R.L. (1990) Max-stable processes and spatial extremes, pre-print. University of North Carolina, USA.

[23] Tiago de Oliveira, J. (1962/63) Structure theory of bivariate extremes, extensions. Est. Mat., Estat. e Econ., 7, 165-195. 

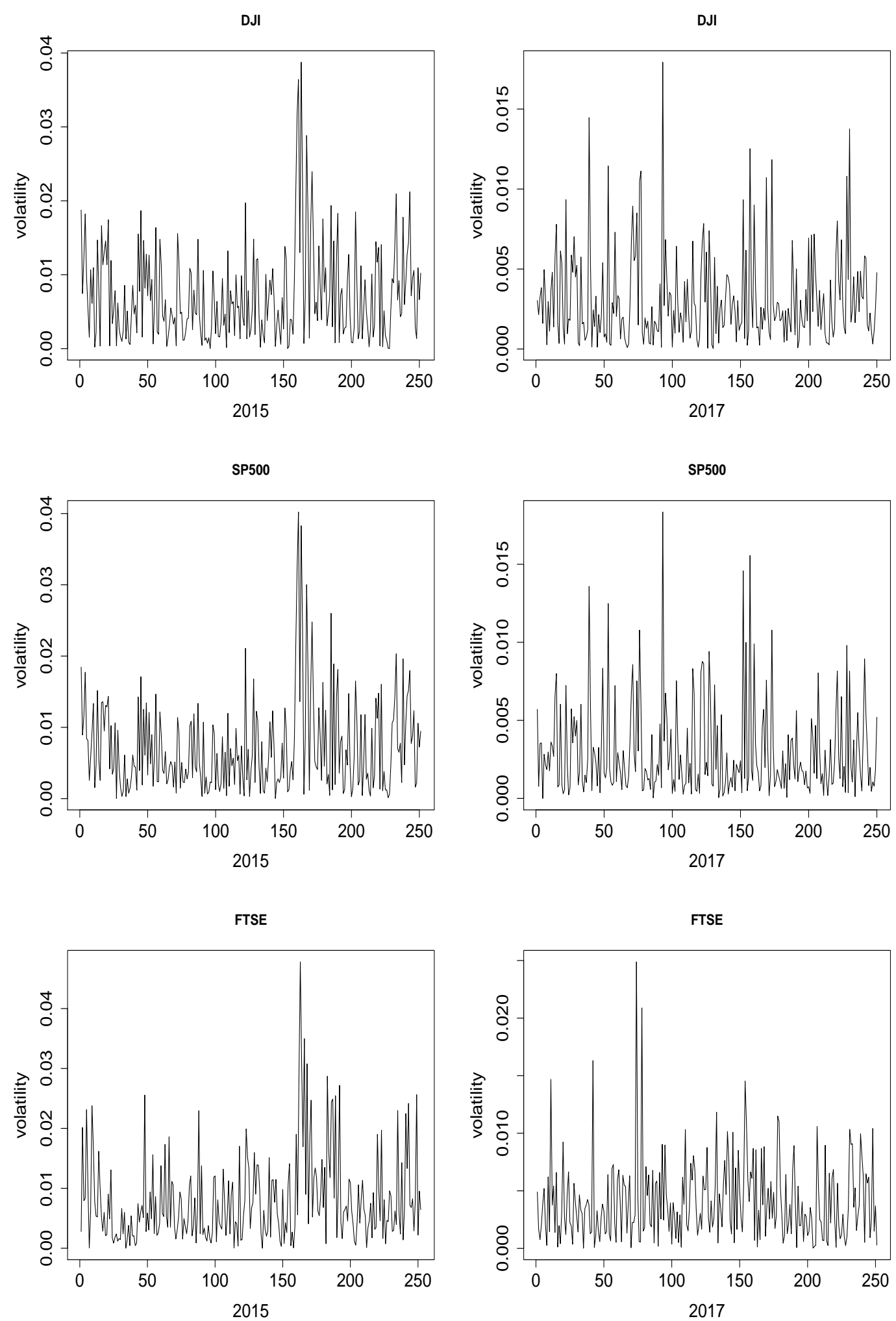

Figure 1: Absolute value of daily closed log-returns (volatility) of DJI, S\&P500 and FTSE in years 2015 (left) and 2017 (right). 